\title{
SHAPE OPERATORS OF EINSTEIN HYPERSURFACES IN INDEFINITE SPACE FORMS
}

\author{
MARTIN A. MAGID
}

\begin{abstract}
The possible shape operators for an Einstein hypersurface in an indefinite space form are classified algebraically. If the shape operator $A$ is not diagonalizable then either $A^{2}=0$ or $A^{2}=-b^{2} I d$.
\end{abstract}

Introduction. In [F] A. Fialkow classifies Einstein hypersurfaces in indefinite space forms, if the shape operator is diagonalizable at each point. He calls such an immersion proper (p. 764). This paper investigates what happens if the immersion is improper, i.e., if the shape operator is not diagonalizable at a point. It is possible for such a shape operator to have complex eigenvalues or eigenvectors with zero length. The main tool is Petrov's classification of symmetric operators in an indefinite inner product space [P].

THEOREM. Let $n>2$. If $f: M^{n} \rightarrow \tilde{M}^{n+1}(\tilde{c})$ is an isometric immersion of an $n$-dimensional indefinite Riemannian manifold into an $n+1$ dimensional space form of constant curvature $\tilde{c}$ and if $M^{n}$ is Einstein, then the shape operator $A_{x}$ at each point $x \in M$ is either diagonalizable or can be put into one of the following two forms.

$$
\begin{aligned}
& A_{x}=\left[\begin{array}{rrrrrrrr}
0 & & & & & & & \\
& \ddots & & & & & & \\
& & 0 & & & & & \\
& & & 0 & \pm 1 & & & \\
& & & & & \ddots & & \\
& & & & & & 0 & \pm 1 \\
& & & & & & 0 & 0
\end{array}\right] \text { or } \\
& A_{x}=\left[\begin{array}{ccccc}
0 & \beta & & & \\
-\beta & 0 & & & \\
& & \ddots & & \\
& & & 0 & \beta \\
& & & -\beta & 0
\end{array}\right]
\end{aligned}
$$

with respect to some specially chosen basis. In the last case $n$ is even and $T_{x}\left(M^{n}\right)$ has signature $(n / 2, n / 2)$.

Received by the editors June $8,1981$.

1980 Mathematics Subject Classification. Primary 53B30. 
Note. The basis in the first case is of the form $\left\{e_{1}, \ldots, e_{p}, l_{1}, \hat{l}_{1}, \ldots, l_{q / 2}, \hat{l}_{q / 2}\right\}$ where $g\left(e_{i}, e_{j}\right)= \pm \delta_{i j}, g\left(e_{i}, l_{j}\right)=0=g\left(e_{i}, \hat{l}_{j}\right)=g\left(l_{i}, l_{j}\right)=g\left(\hat{l}_{i}, \hat{l}_{j}\right)$ and $g\left(l_{j}, \hat{l}_{j}\right)=1$. In the second case the basis is $\left\{e_{1}, f_{1}, e_{2}, f_{2}, \ldots, e_{n / 2}, f_{n / 2}\right\}$ with $g\left(e_{i}, e_{j}\right)=-\delta_{i j}$, $g\left(f_{i}, f_{j}\right)=\delta_{i j}$ and $g\left(e_{i}, f_{j}\right)=0$. This follows from [P].

Preliminaries. The Ricci tensor field $S$ of a manifold $M$ with linear connection is defined by

$$
S(X, Y)=\operatorname{tr}\{V \rightarrow R(V, X) Y\} \quad \text { where } X, Y, V \text { are in } T_{x}(M) .
$$

If $e_{1}, \ldots, e_{n}$ is an orthonormal basis of $T_{x}(M)$, so that $g\left(e_{i}, e_{j}\right)=\sigma_{i} \delta_{i j}$, $\sigma_{i}= \pm 1$, then $S(X, Y)=\sum_{i-1}^{n} \sigma_{i} g\left(R\left(e_{i}, X\right) Y, e_{i}\right)$.

The Gauss equation for a hypersurface in a space form $\tilde{M}(\tilde{c})$ states that

$$
R\left(U_{1}, U_{2}\right) U_{3}=\tilde{c}\left(U_{1} \wedge U_{2}\right) U_{3}+\langle\xi, \xi\rangle\left(A U_{1} \wedge A U_{2}\right) U_{3}
$$

where $R$ is the curvature tensor of the hypersurface, $\xi$ is a local, unit normal and $A$ the shape operator of the isometric immersion.

Thus we see that

$$
\begin{aligned}
S(X, Y)= & \sum_{i=1}^{n} \sigma_{i} g\left(\tilde{c}\left(e_{i} \wedge X\right) Y+\langle\xi, \xi\rangle\left(A e_{i} \wedge A X\right) Y, e_{i}\right) \\
= & \sum_{i=1}^{n} \sigma_{i} \tilde{c}\left[g(X, Y) g\left(e_{i}, e_{i}\right)-g\left(e_{i}, Y\right) g\left(e_{i}, X\right)\right] \\
& +\sum_{i=1}^{n}\langle\xi, \xi\rangle \sigma_{i}\left[g(A X, Y) g\left(A e_{i}, e_{i}\right)-g\left(A X, e_{i}\right) g\left(A Y, e_{i}\right)\right] \\
= & \tilde{c} n g(X, Y)-\tilde{c} \sum_{i=1}^{n} \sigma_{i} g\left(e_{i}, Y\right) g\left(e_{i}, X\right) \\
& +\langle\xi, \xi\rangle \operatorname{tr} A g(A X, Y)-\sum_{i=1}^{n}\langle\xi, \xi\rangle \sigma_{i} g\left(A X, e_{i}\right) g\left(A Y, e_{i}\right) .
\end{aligned}
$$

Note that $\sum_{i=1}^{n} \sigma_{i} g\left(e_{i}, X\right) g\left(e_{i}, Y\right)=g(X, Y)$ so that

$$
S(X, Y)=\tilde{c}(n-1) g(X, Y)+\langle\xi, \xi\rangle\left(\operatorname{tr} A g(A X, Y)-g\left(A^{2} X, Y\right)\right) .
$$

Proof of Theorem. If $M^{n}$ is Einstein then $S(X, Y)=\rho g(X, Y)$. Letting $\langle\xi, \xi\rangle$ $=\tau$ we see then that $[\rho-\tilde{c}(n-1)] I=\tau\left[(\operatorname{tr} A) A-A^{2}\right]$ or $\tau[\rho-\tilde{c}(n-1)] I=$ $(\operatorname{tr} A) A-A^{2}$.

According to Petrov [P] a symmetric operator in an indefinite inner product space can be put into the following form:

$$
A=\left[\begin{array}{cccccc}
B_{1} & & & & & \\
& \ddots & & & & \\
& & B_{k} & & & \\
& & & C_{1} & & \\
& & & & \ddots & \\
& & & & & C_{m}
\end{array}\right]
$$


where

$$
\begin{aligned}
& B_{i}=\left[\begin{array}{ccccc}
d_{i} \lambda_{i} & d_{i} & & & \\
0 & d_{i} \lambda_{i} & d_{i} & & \\
& & & \ddots & \\
& & & & d_{i} \\
& & & & d_{i} \lambda_{i}
\end{array}\right], \quad d_{i}= \pm 1, B_{i} \text { is } s_{i} \times s_{i}, \\
& C_{j}=\left[\begin{array}{ccccccccc}
\alpha_{j} & \beta_{j} & 1 & 0 & & & & & \\
-\beta_{j} & \alpha_{j} & 0 & 1 & & & & & \\
& & \alpha_{j} & \beta_{j} & 1 & 0 & & & \\
& & -\beta_{j} & \alpha_{j} & 0 & 1 & & & \\
& & & & & & \ddots & & \\
& & & & & & & \alpha_{j} & \beta_{j} \\
& & & & & & & -\beta_{j} & \alpha_{j}
\end{array}\right], \quad \beta_{j} \neq 0 \text { and } C_{j} \text { is } 2 t_{j} \times 2 t_{j} \text {. }
\end{aligned}
$$

One computes that

$$
\begin{aligned}
& B_{i}^{2}=\left[\begin{array}{cccccccc}
\lambda_{i}^{2} & 2 \lambda_{i} & 1 & 0 & & \cdots & & 0 \\
0 & \lambda_{i}^{2} & 2 \lambda_{i} & 1 & 0 & \cdots & 0 \\
& & & & & \ddots & & \vdots \\
& & & & \ddots & & 0 \\
& & & & \ddots & & 1 \\
& & & & & & \\
& & & & & & \\
& & & & & & & \lambda_{i}^{2}
\end{array}\right] \text {, } \\
& C_{j}^{2}=\left[\begin{array}{ccccccccc}
\alpha_{j}^{2}-\beta_{j}^{2} & 2 \alpha_{j} \beta_{j} & 2 \alpha_{j} & 2 \beta_{j} & 1 & 0 & & \ldots \\
-2 \alpha_{j} \beta_{j} & \alpha_{j}^{2}-\beta_{j}^{2} & -2 \beta_{j} & 2 \alpha_{j} & 0 & 1 & 0 & \\
0 & 0 & \alpha_{j}^{2}-\beta_{j}^{2} & 2 \alpha_{j} \beta_{j} & 2 \alpha_{j} & 2 \beta_{j} & 1 & 0 & \ldots \\
& & & & & & & & \\
& & & & & & & & \\
& & & & & & & \alpha_{j}^{2}-\beta_{j}^{2}
\end{array}\right] .
\end{aligned}
$$

Letting $\kappa=\tau(\rho-\tilde{c}(n-1))$ we must have $\kappa I=(\operatorname{tr} A) A-A^{2}$.

It is clear from the form of $B_{i}^{2}$ and $C_{j}^{2}$ that $s_{i}<2$ and $t_{j} \leqslant 1$ so that $A$ has blocks of the form

$$
\left[\mu_{i}\right] \text { or }\left[\begin{array}{cc}
d_{j} \lambda_{j} & d_{j} \\
0 & d_{j} \lambda_{j}
\end{array}\right] \text { or }\left[\begin{array}{cc}
\alpha_{k} & \beta_{k} \\
-\beta_{k} & \alpha_{k}
\end{array}\right]
$$


with squares

$$
\left[\mu_{i}^{2}\right] \text { or }\left[\begin{array}{cc}
\lambda_{j}^{2} & 2 \lambda_{j} \\
0 & \lambda_{j}^{2}
\end{array}\right] \text { or }\left[\begin{array}{cc}
\alpha_{k}^{2}-\beta_{k}^{2} & 2 \alpha_{k} \beta_{k} \\
-2 \alpha_{k} \beta_{k} & \alpha_{k}^{2}-\beta_{k}^{2}
\end{array}\right]
$$

By a change of basis $\{l, \hat{l}\} \rightarrow\{-l, \hat{l}\}$ we can assume we have blocks of the form

$$
\left[\mu_{i}\right] \text { or }\left[\begin{array}{cc}
\lambda_{j} & 1 \\
0 & \lambda_{j}
\end{array}\right] \text { or }\left[\begin{array}{cc}
\alpha_{k} & \beta_{k} \\
-\beta_{k} & \alpha_{k}
\end{array}\right]
$$

With trace $A=s$ the equation $s A-A^{2}=\kappa I$ yields

$$
\begin{gathered}
s-2 \lambda_{j}=0, \quad s \beta_{k}-2 \alpha_{k} \beta_{k}=0, \\
s \mu_{i}-\mu_{i}^{2}=\kappa, \quad s \lambda_{j}-\lambda_{j}^{2}=\kappa, \quad s \alpha_{k}-\alpha_{k}^{2}+\beta_{k}^{2}=\kappa .
\end{gathered}
$$

If there are any blocks with $\alpha$ 's and $\beta$ 's, $\beta \neq 0$ so that we have $s / 2=\lambda_{j}, s / 2=\alpha_{k}$, for each $j$ and $k$. Thus all $\lambda_{j}$ 's and $\alpha_{k}$ 's are equal. It is then clear that all $\beta_{k}$ 's are equal. The equations become

(1) $s-2 \lambda=0, s-2 \alpha=0$,

(2) $s \mu_{i}-\mu_{i}^{2}=\kappa, s \lambda-\lambda^{2}=\kappa, s \alpha-\alpha^{2}+\beta^{2}=\kappa$.

Substituting (1) in (2) we have $s \mu_{i}-\mu_{i}^{2}=\kappa, \lambda^{2}=\kappa, \alpha^{2}+\beta^{2}=\kappa$. Since $\lambda=\alpha$ and $\beta \neq 0$, there can be blocks with $\alpha$ 's or blocks with $\lambda$ 's but not both. In either case we have

$$
\mu_{i}=\frac{1}{2}\left(s \pm \sqrt{s^{2}-4 \kappa^{2}}\right)
$$

If $\kappa=\lambda^{2}, \mu_{i}=s / 2$. If $\kappa=\alpha^{2}+\beta^{2}, s^{2}-4 \kappa^{2}<0$ and there are no $\mu_{i}$ 's.

If there is a block with a $\lambda$, then $\lambda=s / 2$ and $\mu_{i}=s / 2$, for each $i$. If $p$ is the number of $\mu$ 's which appear in $A$ and $2 q$ the number of $\lambda$ 's

$$
s=p \mu+2 q \lambda=p(s / 2)+2 q(s / 2) .
$$

Thus $s(1-p / 2-q)=0$. But $p+2 q>3$, so $s=0$. One possibility for $A$ then is

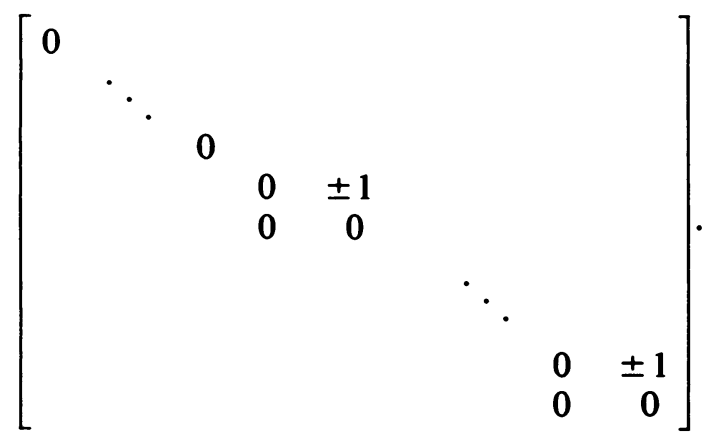


If there is a block with a $\beta$, there are no other types of blocks. Since $\alpha=s / 2$ we again see that $s=0$ and

$$
A=\left[\begin{array}{ccccc}
0 & \beta & & & \\
-\beta & 0 & & & \\
& & \ddots & & \\
& & & 0 & \beta \\
& & & -\beta & 0
\end{array}\right]
$$

Q.E.D.

These shape operators all occur in examples of Einstein hypersurfaces in indefinite space forms.

EXAMPLE 1. $\mathbf{R}_{n}^{2 n} \rightarrow \mathbf{R}_{n}^{2 n+1}$.

$$
\begin{aligned}
\left(x_{1}, \ldots, x_{2 n-1}, x_{2 n}\right) & \mapsto\left(x_{1}+x_{2}, x_{3}+x_{4}, \ldots, x_{2 n-1}+x_{2 n},\right. \\
& \left.x_{1}-x_{2}, \ldots, x_{2 n-1}-x_{2 n}, x_{2}^{2}+x_{4}^{2}+\cdots+x_{2 n}^{2}\right) .
\end{aligned}
$$

The ambient space has the standard inner product $(-, \ldots,-,+\cdots+)$ with $n$ negative signs. The shape operator is

$$
\left[\begin{array}{lllll}
0 & 1 & & & \\
0 & 0 & & & \\
& & \ddots & & \\
& & & 0 & 1 \\
& & & 0 & 0
\end{array}\right]
$$

at each point.

EXAMPLE 2. $\operatorname{CS}^{n}(1)=\left\{\left(Z_{1}, \ldots, Z_{n+1}\right) \in \mathbf{C}^{n+1}: Z_{1}^{2}+\cdots+Z_{n+1}^{2}=1\right\}$ in $S_{n+1}^{2 n+1}$ has shape operator

$$
\left[\begin{array}{ccccc}
0 & 1 & & & \\
-1 & 0 & & & \\
& & \ddots & & \\
& & & 0 & 1 \\
& & & -1 & 0
\end{array}\right]
$$

at each point.

Applications. This allows us to obtain some information about isometric immersions of Einstein hypersurfaces.

Proposition. If $f: M^{2 n} \rightarrow \tilde{M}^{2 n+1}(\tilde{c})$ is an isometric immersion of an Einstein manifold and if $A_{x}$ is not diagonalizable at each point then $A^{2}=0$ everywhere or $A^{2}=-b^{2} I$ everywhere, for $b$ a nonzero constant.

Proof. If $A_{x}$ is not diagonalizable then the proof of the theorem shows $\operatorname{tr} A_{x}=$ 0. Thus

$$
\kappa I-\left(\operatorname{tr} A_{x}\right) A_{x}+A_{x}^{2}=0=\kappa I+A_{x}^{2}
$$

for $\kappa$ a constant. The proof also shows $\kappa \geqslant 0$. 
Proposition. If $f: M^{2 n} \rightarrow \tilde{M}^{2 n+1}(\tilde{c})$ is an isometric immersion of an Einstein manifold with $A_{x}^{2}=0, \operatorname{rank} A_{x}=n$ for all $x \in M^{2 n}$, then $\operatorname{ker} A$ is a smooth, integrable, totally geodesic, and totally degenerate n-dimensional distribution on $M$.

Proof. See also [G]. Choose $U_{1}, \ldots, U_{n}$ at $p$ such that $A U_{j} \neq 0$ and $U_{1}, \ldots, U_{n}$ are linearly independent. Then in a neighborhood of $p, A U_{j} \neq 0$. Since $A A U_{j}=0$, $A U_{1}, \ldots, A U_{n}$ form a basis for $\operatorname{ker} A$ in a neighborhood of $p$ and $\operatorname{ker} A$ is a smooth, $n$-dimensional distribution.

If $X, Y \in \operatorname{ker} A$ we have, by Codazzi's equation that $A\left(\nabla_{X} Y\right)-\nabla_{X}(A Y)=$ $A\left(\nabla_{Y} X\right)-\nabla_{Y}(A X)$ so

$$
A\left(\nabla_{X} Y\right)-A\left(\nabla_{Y} X\right)=0, \quad A[X, Y]=0
$$

and ker $A$ is integrable.

It is easy to see that $A^{2}=0, \operatorname{rank} A=n$ implies that $\operatorname{ker} A=\operatorname{im} A$. If $U, V \in$ $T_{x} M,\langle A U, A V\rangle=\left\langle A^{2} U, V\right\rangle=0$ so that $\operatorname{ker} A$ is totally degenerate, i.e., has no metric.

Finally, if $X, Y \in \operatorname{ker} A$, then $\nabla_{X} Y \in \operatorname{ker} A .\langle Y, A U\rangle=0$ so

$$
\begin{aligned}
X\langle Y, A U\rangle & =\left\langle\nabla_{X} Y, A U\right\rangle+\left\langle Y, \nabla_{X}(A U)\right\rangle \\
& =\left\langle\nabla_{X} Y, A U\right\rangle+\left\langle Y, \nabla_{U}(A X)\right\rangle+\langle Y, A[U, X]\rangle=\left\langle\nabla_{X} Y, A U\right\rangle,
\end{aligned}
$$

since $A X=A Y=0$. Thus $A\left(\nabla_{X} Y\right) \perp U$ for all $U$ and $A\left(\nabla_{X} Y\right)=0$.

Note. In a subsequent paper [M], I classified Einstein hypersurfaces with $A^{2}=$ $-b^{2}$ Id. They are certain complex spheres, of which Example 2 is one.

\section{BIBLIOGRAPHY}

[F] A. Fialkow, Hypersurfaces of a space of constant curvature, Ann. of Math. 39 (1938), 762-785.

[G] L. Graves, Codimension-one isometric immersions between Lorentz spaces, Thesis, Brown University, 1977.

[M] M. Magid, Indefinite Einstein hypersurfaces (preprint).

[P] A. Z. Petrov, Einstein spaces, Pergamon Press, Hungary, Oxford and New York, 1969.

Department of Mathematics, Wellesley College, Wellesley, Massachusetts 02181

Current address: Department of Mathematics, University of California, Berkeley, California 94720 